\title{
Un grand Ami nous a quittés... Maurice Gariel (1884-1960)
}

Maurice Gariel, Officier de la Légion d'Honneur, Croix de guerre, Président-Directeur Général des Etablissements Neyrpic, est mort à Grenoble le 22 janvier 1960. Avec lui, l'Hydraulique perd l'un de ses pionniers les plus brillants, l'Industrie française l'un de ses chefs les plus éminents et les plus dynamiques, La Houille Blanche le meilleur des conseillers et le plus précieux des amis.

Né à Grenoble en 1884, licencié ès-Sciences, Ingénieur de l’Ecole Supérieure d'Electricité, Maurice Gariel était entré en 1906 à la Maison Neyret-Beylier, que son histoire, vieille déjà de cinquante ans, et ses plus belles réalisations associaient étroitement au magnifique essor de la «houille blanche », héritière des moulins de jadis et du génie de Bergès, chargée des premiers fruits de son alliance avec la toute jeune électricité.

Maurice Gariel fit connaissance avec l'hydraulique en bataillant contre les régulateurs des premières grandes centrales hydroélectriques. Et déjà sa manière apparaît, faite à la fois d'une intuition toujours à l'affût, et du souci de rassembler et d'analyser minutieusement les moindres éléments de chaque problème. A cette période se rattachent ses premières publications, en particulier «Etude théorique et expérimentale des coups de bélier dans les conduites forcées » (1919), en collaboration avec Camichel et Eydoux; «Traité des turbines hydrauliques» (1926) en collaboration avec Rateau et Eydoux.

Fidèle au souvenir de ses premières joutes hydrauliques, il marqua très profondément, tout au long de sa carrière, les travaux menés sous sa direction en vue de mieux faire et de mieux diriger les régulateurs, sur lesquels il ironisait volontiers, proclamant qu'ils n'avaient en cinquante ans guère gagné en docilité, mais que l'interconnexion était venu tout arranger...

1914; Maurice Gariel est grièvement blessé dès les premiers combats dans les Vosges. Le voilà de retour à Grenoble, et le voilà, en 1917, en pleine guerre, chargé de créer une nouvelle usine. Il fallait donner, à la construction des turbines hydrauliques, l'impulsion nouvelle qu'appelait l'accroissement accéléré des besoins en énergie de l'industrie française.

Neyrpic était né. En quarante-trois ans, Maurice Gariel n'en fit pas seulement l'un des grands constructeurs mondiaux; il y suscita, il y dirigea, il y développa tout un faisceau d'études, de recherches, d'inventions révélant peu à peu, à la vieille hydraulique, des perspectives insoupçonnées.

Certes, les turbines hydrauliques eurent elles-mêmes leur part, toujours très généreuse, des recherches poursuivies. Maurice Gariel a suivi pas à pas, bien souvent orienté et dirigé toute l'évolution qui a hissé les turbines hydrauliques jusque vers les sommets à 150000 ou $200000 \mathrm{ch}$ de puissance unitaire couramment atteints aujourd'hui. Plus direct, plus déterminant encore peut-être, se révèle le rôle qu'il a joué dans l'amélioration des conditions d'équipement des basses chutes. La récente mise au point des groupes «bulbe » doit incontestablement être portée au crédit de son action personnelle, fondée une fois de plus sur l'intuition très claire, et très précise, des possibilités à escompter comme des difficultés à surmonter. Machines de plus en plus puissantes, équipement de plus en plus rationnel des basses chutes, ce double souci, cette double orientation de l'action de Maurice Gariel trouvent leur consécration dans deux des événements qui, sur le plan technique, ont marqué les derniers mois de sa vie : l'implantation, dans ses ateliers de Grenoble, d'un nouveau tour vertical de très grande puissance, adapté à l'échelle des réalisations à venir; la mise en route à Saint-Malo, avec un remarquable succès, du groupe «bulbe » prototype de l'usine marémotrice de la Rance, groupe à turbo-pompage amont-aval ou aval-amont, aboutissement de plus de quinze années de recherches acharnées, menées sous sa direction très vigilante et très active.

Mais l'art des turbines, ce fut aussi pour Maurice Gariel, ce fut surtout peut-être, sur les chemins de l'hydraulique, le carrefour de la similitude. Depuis le début du siècle, sous l'impulsion décisive de Rateau, la similitude des turbo-machines était à l'ordre du jour. Elle portait partout ses fruits, et à Grenoble même, à la Maison Neyret-Beylier, puis aux nouvelles usines Neyrpic, les stations d'essais de turbines sur modèles réduits se multipliaient, se perfectionnaient, prêtes à jouer leur rôle aujourd'hui 
capital dans le raffinement des tracés et des performances. Rompu à cette technique, émerveillé de ses résultats, Maurice Gariel eut cette intuition géniale que l'hydraulique offrait ainsi, à qui saurait le saisir et l'exploiter, un talisman aux prodigieuses vertus. Armé de ce talisman, fort de son intuition et de son enthousiasme, il partit à l'aventure.

Très vite l'aventure se dessina : il ne s'agissait pas moins que d'une conquête de tous les domaines de l'Hydraulique. Au départ, en 1923, ce fut le modèle réduit de la chambre d'eau de la centrale de Drac-Romanche, près de Grenoble. Aujourd'hui, ce sont des ha de modèles, c'est la ruche bourdonnante de la Sogréah et de ses 500 ingénieurs et techniciens, c'est le monde entier et toute l'hydraulique, celle que l'on connaissait, celle que l'on soupçonnait, celle que l'on n'attendait pas. A sa suite, Maurice Gariel a entraîné toute une pléiade de chercheurs, une immense équipe sans équivalent au monde; que de fois, aussi dans le dédale de ses laboratoires, il a entraîné ses amis, ses visiteurs, spécialistes ou profanes, leur découvrant avec une éblouissante virtuosité la trame cachée, devenue soudain évidente, de tout un monde de déversements, de débordements et de tempêtes dûment dirigées, mesurés ou apaisés. Toute l'hydraulique y passait : celle des barrages, celle des fleuves, celle de la mer qui lui était si chère, celle des suspensions, des décantations, des triages, celle de l'acier, celle, si paradoxale, des flammes, celle vitale des grands aménagements hydro-agricoles, celle d'hier qu'il racontait si bien, celle de demain qu'il pressentait avec tant d'audace et de lucidité.

Car Maurice Gariel n'était pas seulement un technicien et un entraîneur, c'était un apôtre convaincu que toute conquête scientifique ou technique ne trouve son plein épanouissement que dans une très large et très généreuse diffusion.

Tel est bien l'esprit dans lequel il apportait son concours sans réserve, toujours décisif, à un grand nombre d'institutions, d'organismes nationaux ou internationaux, officiels ou non, favorisant cette diffusion. A Grenoble, il fut d'un des précurseurs d'abord, l'un des principaux artisans ensuite, de cette liaison Université-Industrie, considérée un peu partout, aujourd'hui, comme l'une des bases d'un développement harmonieux de la recherche scientifique et de la technique industrielle. Membre du Conseil de l'Université de Grenoble, Président du Comité de Patronage des Etudiants Etrangers, il manifesta aussi le prix qu'il attachait au rayonnement de l'Université, et aux courants d'échanges internationaux dont elle pouvait être le point de convergence.

Très éloquent enfin est l'appui qu'il ne cessa d'apporter pendant plus de quarante ans à la Société Hydrotechnique de France. Membre de son Comité Technique depuis 1917, il apporta une collaboration assidue et efficace à plusieurs de ses sections de travail, en particulier à la section «Machines» qu'il fut appelé à présider en 1948, à la suite du regretté Louis Bergeron. Enfin le Conseil d'administration de la Société Hydrotechnique de France, dont il était membre depuis 1934, devait le porter par deux fois à sa présidence de 1950 à 1952, et de 1956 à 1958. A ce titre, il présida, avec sa maîtrise et sa. virtuosité coutumières, à la préparation et au déroulement des deux très belles manifestations que furent : - les Deuxièmes Journées de l'Hydraulique, à Grenoble, en 1952;

- les Quatrièmes Journées de l'Hydraulique, à Aix-en-Provence en 1958.

Sur cet aspect si attachant et si marquant de la personnalité de Maurice Gariel, il y aurait beaucoup à dire encore. Mais, comment pourrions-nous ici ne pas évoquer, avec une profonde reconnaissance et une vive émotion, le conseiller incomparable, l'ami très fidèle et très précieux de notre Revue. Lorsqu'en 1945 notre Directeur Général, Pierre Danel, ayant formé le projet de créer une grande revue internationale d'hydraulique s'en ouvrit à Maurice Gariel, les encouragements et l'appui qu'il trouva auprès de lui se révélèrent décisifs. Depuis, Maurice Gariel a suivi de très près tous nos efforts, tous nos succès, toutes nos difficultés. Lorsqu'il apparut nécessaire de donner à notre Revue les assises correspondant à sa mission, c'est à son action personnelle, à son autorité que revint, pour une large part, le mérite de la création de l'Association pour la Diffusion de la Documentation Hydraulique, sous l'égide de laquelle nous travaillons encore aujourd'hui, et qu'il présidait depuis plusieurs années.

Maurice Gariel, notre ami, notre conseiller, était réellement notre guide, un guide irremplaçable dont nous portons douloureusement et respecteusement le deuil en union avec sa famille, ses collaborateurs et ses innombrables amis dans le monde entier.

Technicien doué d'une infaillible intuition, entraîneur irrésistible rompu à la maîtrise des choses et à la conduite des hommes, chef d'industrie au sens le plus élevé et le plus complet du terme, Maurice Gariel laissera par-dessus tout, à ceux qui eurent l'honneur et le bonheur de l'approcher et de travailler à ses côtés, le souvenir et l'exemple de l'amour que, de tout son cœur, de toute son intelligence, il portait aux hommes, à leur misère, à leur faiblesse, mais à leur grandeur et à leur génie aussi, dans tous les domaines de la pensée, de la science et des arts; amour souriant, amour rayonnant, puisé à la source très pure et intarissable de l'ardente foi qui l'animait. 